\title{
Cardiac Dysfunction in Critically III patients with COVID-19 - A Multicentre Observational Study
}

Jacob Holmqvist

Sahlgrenska University Hospital: Sahlgrenska universitetssjukhuset Josefine Beck-Friis

Sahlgrenska University Hospital: Sahlgrenska universitetssjukhuset

Carl Jensen

NU Hospital Group: NU-sjukvarden

Keti Dalla

Sahlgrenska University Hospital Mölndal Hospital: Molndals Sjukhus

Simon Mårdstam

Södersjukhuset

Jens Christensen

Södersjukhuset

Nina Nordén

Sahlgrenska University Hospital Mölndal Hospital: Molndals Sjukhus

Elin Rosén-Wetterholm

Goteborgs universitet Sahlgrenska Akademin

\section{Oscar Cavefors}

Sahlgrenska University Hospital: Sahlgrenska universitetssjukhuset

\section{Aylin Yilmaz}

Sahlgrenska University Hospital: Sahlgrenska universitetssjukhuset

\section{Maria Cronhjort}

Södersjukhuset

\section{Björn Redfors}

Sahlgrenska University Hospital: Sahlgrenska universitetssjukhuset

Jonatan Oras ( $\nabla$ jonatan.oras@gu.se)

Goteborgs universitet Institutionen for kliniska vetenskaper https://orcid.org/0000-0001-8890-6752

\section{Research}

Keywords: COVID-19, cardiac dysfunction, echocardiography, cardiac biomarkers, intensive care unit

Posted Date: January 5th, 2021 
DOl: https://doi.org/10.21203/rs.3.rs-138541/v1

License: (c) (1) This work is licensed under a Creative Commons Attribution 4.0 International License. Read Full License 


\section{Abstract}

Introduction: The importance of cardiac dysfunction in critically ill patients with COVID-19 is not well studied. The aim of the study was to assess the incidence, clinical risk factors, and prognosis of cardiac dysfunction in critical illness caused by COVID-19, and to evaluate if cardiac biomarkers can detect this condition.

Methods: This was a multicentre observational study performed in five intensive care units (ICUs) in Sweden. Patients admitted to participating ICU with COVID-19 were examined with echocardiography within 72 hours from admission to the ICU and again after four to seven days. Cardiac biomarkers and clinical data were collected at the time of echocardiography. Cardiac dysfunction was defined as either left ventricular (LV) dysfunction (having an ejection fraction $<50 \%$ and/or regional hypokinesia) or right ventricular (RV) dysfunction (having a tricuspid annular plane systolic excursion (TAPSE) $<17 \mathrm{~mm}$ or a moderate/severe RV dysfunction assessed visually).

Results: We included 132 patients of whom 94 (71\%) were included prospectively. The vast majority were intubated $(n=127)$. At the time of admission to ICU, $35(27 \%)$ patients had cardiac dysfunction and 7 patients (5\%) had cardiac dysfunction detected later in the ICU-period. LV dysfunction was found in 18 patients and RV dysfunction in 17 patients, 7 patients had both RV and LV dysfunction. Noradrenaline > $0.20 \mu \mathrm{g} / \mathrm{kg} / \mathrm{min}$ was the only clinical variable associated with a higher risk of cardiac dysfunction. RV dysfunction was associated with an increased risk of death in a risk-adjusted model (OR 3.98, $p=0.013$ ). Troponin and N-terminal pro b-type natriuretic peptide (NTproBNP) had moderate values in detecting cardiac dysfunction (AUC 0.729 and AUC 0.744 , respectively). A combination of troponin $<1.44$ times the upper reference limit and NTproBNP $<857 \mathrm{ng} / \mathrm{L}$ had $85 \%$ probability of excluding cardiac dysfunction.

Conclusions: Cardiac dysfunction is common in critically ill patients with COVID-19. Although not easily detected with clinical variables, cardiac biomarkers might be helpful. RV dysfunction is associated with an increased risk of death, these patients might benefit from further investigation or treatments.

Trial registration: Registered on 24 Aug 2020 at Clinicaltrials.gov;registration number NCT04524234.

\section{Background}

Coronavirus disease 2019 (COVID-19) is caused by severe acute respiratory syndrome coronavirus 2 (SARS-CoV-2). As of 21 December 2020, more than 70 million cases have been diagnosed worldwide and 1.6 million people have died from the disease [1]. The clinical characteristics of COVID-19 range from asymptomatic to critical infection (respiratory failure, shock, or multiorgan dysfunction), affecting around $5 \%$ of cases [2, 3]. Acute respiratory distress syndrome (ARDS) is the main complication. Other major complications in patients requiring intensive care and mechanical ventilation are cardiac injury and arrythmias [4]. 
Myocardial injury with elevated troponin or creatine kinase is reported in $12-38 \%$ of hospitalized patients with COVID-19 [5, 6] and in 59\% of patients treated in ICUs [7]. Cardiac complications, on a case report basis, include myocarditis [8-10], COVID-19 associated Takotsubo cardiomyopathy [11, 12], and myocardial infarction [12]. Several studies have reported abnormalities in left and right ventricular function in a large proportion of patients with COVID-19 undergoing echocardiography [12-15]. Studies have also shown that left and right ventricular dysfunction are independently correlated to increased mortality in patients with COVID-19 $[14,16]$. Few prospective studies of critically ill patients with COVID19 undergoing echocardiography exist and are limited in scope $[13,17,18]$. Thus, the clinical importance of cardiac dysfunction in critical illness caused by COVID-19 is not yet established. In addition, studies linking echocardiographic findings with clinical data, cardiac biomarkers, and mortality are lacking.

The aim of the present study was to describe the incidence, risk factors, and mortality related to cardiac dysfunction in critical illness caused by COVID-19, and to assess the value of biomarkers for the detection of this condition.

\section{Methods}

This was a multicentre observational study. It was approved by the Swedish Ethics Review Authority Sweden (approval number 036 - 18, 2020 - 01684 and 2020-03815) and registered in the international database at Clinicaltrials.gov (registration number NCT04524234).

\section{Study design and inclusion}

The study was performed at five ICUs in Sweden from 20 April to 30 August 2020 (details of each participating ICU, the ICU settings, and principles of patient treatment are described under Supplemental data). Most patients were included prospectively $(n=94,71 \%)$, however 30 patients were included retrospectively from one of the five study sites. In addition, nine patients who had followed the study protocol before the ethics review was approved were included retrospectively from the other four centres.

All patients $\geq 18$ years of age who were admitted to a participating ICU with verified COVID-19 were eligible for inclusion. Entering patients had an echocardiogram performed within 72 hours from admission to the ICU. Echocardiography was repeated in four to seven days as study resources became available or if there was evidence of clinical deterioration. Cardiac biomarkers, routine laboratory tests, and clinical data were recorded at each exam. Consent to be included in the study was obtained from the patient or patient's next of kin whenever possible. Retrospective inclusion of patients who had followed the study protocol was approved by the ethics review authority, and for these patients consent was waived.

\section{Echocardiography, definitions, recordings, and measurements}


Echocardiography was performed according to standard protocol (see Supplemental data) for assessment of left ventricular (LV) or right ventricular (RV) dysfunction and estimation of pulmonary artery pressure (PAP). Tissue Doppler was not available in all centres and this modality was not further investigated. Cardiac dysfunction was defined as having either LV or RV dysfunction. LV dysfunction was defined as ejection fraction $<50 \%$ with or without regional hypokinesia assessed with eyeballing. RV dysfunction was defined as having a tricuspid annular plane systolic excursion (TAPSE) $<17 \mathrm{~mm}$ or a moderately or severely depressed RV dysfunction qualitatively judged visually. Elevated PAP was defined as jet velocity of a tricuspid regurgitation $>2.9 \mathrm{~m} / \mathrm{s}$ (TR Vmax), or, in patients with no tricuspid regurgitation present, having indirect signs of elevated PAP such as RV dilation and a pulmonary acceleration time $<100 \mathrm{~ms}[19,20]$. Velocity Time Integral (VTI), area of LV outflow tract (LVOT) with calculation of stroke volumes, and cardiac output were assessed, wherever image quality was acceptable. Examinations were recorded and, reviewed by a physician certified in echocardiography, if performed by a non-certified intensivist.

At the time of echocardiography, blood samples for measurement of the cardiac biomarkers troponin and $\mathrm{N}$-terminal pro b-type natriuretic peptide (NTproBNP) were obtained and clinical data was recorded. Four sites used highly sensitive troponin $\mathrm{T}$ and one site used troponin I. The levels are presented as times above upper limit of normal. The following clinical data was recorded on admission: age, sex, medical history, and Simplified Acute Physiology Score 3 (SAPS 3) [21]. Along with each echocardiography we also recorded systolic blood pressure, mean arterial pressure, diastolic blood pressure, noradrenaline dosage, lactate levels, respiratory settings, and $\mathrm{PaO}$ /FiO2-ratio. Routine laboratory tests, including Creactive protein (CRP), leukocyte count, creatinine levels, and d-dimer, were obtained on the same day as echocardiography. Mortality at 30 days and death occurring within 90 days of admission were registered.

\section{Pre-defined outcomes}

The pre-defined primary outcome was 30-day mortality in patients with, versus those without, cardiac dysfunction on admission. Sub-group analysis was performed for LV and RV dysfunction. Pre-defined secondary outcomes were a) incidence of LV and RV dysfunction within 72 hours of admission and during ICU stay; b) clinical risk factors associated with cardiac dysfunction; and c) levels of the cardiac biomarkers troponin and NTproBNP in patients with, as opposed to without cardiac dysfunction. Other analyses were not pre-defined at the initiation of the study and are considered exploratory.

\section{Statistics}

A statistical analysis plan was written before the analyses were performed. Continuous variables were checked for normality. Normally distributed variables are presented as mean \pm standard deviation; nonnormally distributed variables are presented as median and interquartile range (IQR). Student's T-test was used to compare means of normally distributed variables and the Mann-Whitney $U$ test was used for comparison of distributions of non-normally distributed variables. Fisher's exact test was used for comparison of binary outcomes between two groups. A generalized estimating equation was used for identification of clinical variables associated with an increased risk of experiencing cardiac dysfunction 
at any time in the ICU. Logistic regression was used to evaluate mortality at 30 days between patients with or without cardiac dysfunction in a non-adjusted and a risk-adjusted analysis (primary outcome). Kaplan-Meier methodology with a log rank test were used to compare incidences over time. Receiver operating characteristics (ROC) analyses were used for estimation of sensitivity and specificity of troponin and NTproBNP for detection of cardiac dysfunction. IBM SPSS Statistics Version 26 were used for the statistical analyses.

No power analysis was performed, as little data of the study population was available when the study was initiated but we aimed for an inclusion of at least 100 patients.

\section{Results}

Out of 344 patients admitted to the participating ICUs during the study period, 137 were included in the study. Inclusion failure was mainly because no investigator involved in the study was available for an inclusion or echocardiographic examination. In a sensitivity analysis regarding SAPS score and age, the study population was representative of each ICU's patient population [22]. Two patients were excluded due to their echocardiography being performed $>72$ hours from admission. An additional three patients were excluded as a result of poor echocardiographic image quality. Thus, a total of 132 patients included in the final analysis (Fig. 1).

\section{Study population}

The median age of the cohort was 63 years (IQR 53-70); 34 (26\%) were women. Median body mass index (BMI) was 30 (IQR 25-33). Seventeen patients (13\%) had a history of cardiac disease and 74 others $(56 \%)$ had a risk factor for cardiovascular disease. A history of coronary artery disease, valvular disease, or hypertension was more common in patients with cardiac dysfunction. The mean SAPS score was $52 \pm 9$ points (Table 1 ). A total of 127 patients (96\%) were intubated during their ICU stay. Patients were given low-molecular weight heparine (LMWH) in doses corresponding to 0.77 (IQR 0.61-0.95) times the treatment dose for acute thrombotic disease. 
Table 1

Background data

\begin{tabular}{|c|c|c|c|c|c|}
\hline \multirow[t]{2}{*}{ Category } & \multirow[t]{2}{*}{ Variable } & \multirow{2}{*}{$\begin{array}{l}\text { All patients } \\
(n=132)\end{array}$} & \multicolumn{3}{|c|}{ Cardiac function } \\
\hline & & & $\begin{array}{l}\text { Normal } \\
\text { function ( } n= \\
90)\end{array}$ & $\begin{array}{l}\text { Cardiac } \\
\text { dysfunction ( } n= \\
\text { 42) }\end{array}$ & $\begin{array}{l}\mathrm{p} \text { - } \\
\text { value }\end{array}$ \\
\hline \multirow[t]{4}{*}{ Demographics } & Age, years & $63(53-70)$ & $64(53-70)$ & $61(52-68)$ & 0.767 \\
\hline & Women, n (\%) & $34(26)$ & $27(30)$ & $7(17)$ & 0.135 \\
\hline & $\mathrm{BMI}$ & $30(25-33)$ & $30(26-33)$ & $28(25-34)$ & 0.577 \\
\hline & SAPS3 & $51 \pm 9$ & $51 \pm 9$ & $53 \pm 10$ & 0.285 \\
\hline Medical & $\begin{array}{l}\text { Any cardiac } \\
\text { disease, n (\%) }\end{array}$ & $17(13)$ & $8(9)$ & $9(21)$ & 0.055 \\
\hline \multirow[t]{13}{*}{ history } & Heart failure, n (\%) & $0(0)$ & $0(0)$ & $0(0)$ & - \\
\hline & $\begin{array}{l}\text { Coronary artery } \\
\text { disease, } \mathrm{n}(\%)\end{array}$ & $15(11)$ & $8(9)$ & $7(17)$ & 0.240 \\
\hline & $\begin{array}{l}\text { Atrial fibrillation, } \mathrm{n} \\
(\%)\end{array}$ & $5(4)$ & $2(2)$ & $3(7)$ & 0.326 \\
\hline & $\begin{array}{l}\text { Valvular disease, } \mathrm{n} \\
(\%)\end{array}$ & $2(2)$ & $0(0)$ & $2(5)$ & 0.100 \\
\hline & $\begin{array}{l}\text { Other cardiac } \\
\text { disease, n (\%) }\end{array}$ & $1(1)$ & $0(0)$ & $1(2)$ & 0.318 \\
\hline & $\begin{array}{l}\text { Cerebrovascular } \\
\text { disease, } \mathrm{n}(\%)\end{array}$ & $10(8)$ & $5(6)$ & $5(12)$ & 0.288 \\
\hline & $\begin{array}{l}\text { COPD or astma, } \mathrm{n} \\
(\%)\end{array}$ & $13(10)$ & $9(10)$ & $4(10)$ & $>0.999$ \\
\hline & Renal failure, $\mathrm{n}(\%)$ & $7(5)$ & $4(4)$ & $3(7)$ & 0.679 \\
\hline & $\begin{array}{l}\text { Any risk factor of } \\
\text { CVD, } n(\%)\end{array}$ & $74(56)$ & $44(49)$ & $30(71)$ & 0.023 \\
\hline & Diabetes, n (\%) & $29(22)$ & $19(21)$ & $10(24)$ & 0.822 \\
\hline & Hypertension, n (\%) & $58(44)$ & $32(36)$ & $26(62)$ & 0.005 \\
\hline & $\begin{array}{l}\text { Hyperlipidemia, } n \\
(\%)\end{array}$ & $25(19)$ & $13(14)$ & $12(29)$ & 0.061 \\
\hline & $\begin{array}{l}\text { Thrombotic } \\
\text { disease, } \mathrm{n}(\%)\end{array}$ & $6(5)$ & $4(4)$ & $2(5)$ & $\begin{array}{l}> \\
0.999\end{array}$ \\
\hline
\end{tabular}

$\mathrm{BMI}=$ body mass index, SAPS3 = simplified acute physiology score $3, \mathrm{COPD}=$ chronic obstructive pulmonary disease, RAAS-blockers $=$ renin angiotensin aldosterone system blockers 


\begin{tabular}{|c|c|c|c|c|c|}
\hline \multirow[t]{2}{*}{ Category } & \multirow[t]{2}{*}{ Variable } & \multirow{2}{*}{$\begin{array}{l}\text { All patients } \\
(n=132)\end{array}$} & \multicolumn{3}{|c|}{ Cardiac function } \\
\hline & & & $\begin{array}{l}\text { Normal } \\
\text { function }(n= \\
90)\end{array}$ & $\begin{array}{l}\text { Cardiac } \\
\text { dysfunction }(n= \\
42)\end{array}$ & $\begin{array}{l}\mathrm{p} \text { - } \\
\text { value }\end{array}$ \\
\hline & Other, n (\%) & $28(21)$ & $18(20)$ & $10(24)$ & 0.651 \\
\hline \multirow[t]{7}{*}{ Medications } & $\begin{array}{l}\text { RAAS-blockers, n } \\
(\%)\end{array}$ & $40(30)$ & $23(26)$ & $17(40)$ & 0.104 \\
\hline & Betablockers, n (\%) & $31(23)$ & $20(22)$ & $11(26)$ & 0.662 \\
\hline & $\begin{array}{l}\text { Platelet inhibitors, } \\
\mathrm{n}(\%)\end{array}$ & $24(18)$ & $13(14)$ & $11(26)$ & 0.145 \\
\hline & $\begin{array}{l}\text { Other } \\
\text { anticoagulants, } \mathrm{n} \\
(\%)\end{array}$ & $5(4)$ & $3(3)$ & $2(5)$ & 0.653 \\
\hline & $\begin{array}{l}\text { Other } \\
\text { antihypertensives, } \\
\mathrm{n}(\%)\end{array}$ & $32(24)$ & $20(22)$ & $12(29)$ & 0.514 \\
\hline & $\begin{array}{l}\text { Anti-diabetics, n } \\
(\%)\end{array}$ & $20(15)$ & $12(13)$ & $8(19)$ & 0.439 \\
\hline & $\begin{array}{l}\text { Other medications, } \\
\mathrm{n}(\%)\end{array}$ & $63(47)$ & $42(32)$ & $21(50)$ & 0.700 \\
\hline $\begin{array}{l}\mathrm{BMI}=\text { body } \mathrm{m} \\
\text { pulmonary } \mathrm{d}\end{array}$ & $\begin{array}{l}\text { ndex, SAPS3 = simp } \\
\text { e, RAAS-blockers = r }\end{array}$ & $\begin{array}{l}\text { ed acute ph } \\
n \text { angiotens }\end{array}$ & $\begin{array}{l}\text { ology score } 3 \\
\text { Idosterone sy }\end{array}$ & $\begin{array}{l}\mathrm{D}=\text { chronic obstr } \\
\text { blockers }\end{array}$ & \\
\hline
\end{tabular}

Median time to first echocardiography was one day (IQR 1-2); median time to second echocardiography was seven days (IQR 4-9). Thirty-five patients (26\%) had cardiac dysfunction upon admission to the ICU, of whom 17 (14\%) had LV dysfunction, 16 (12\%) had RV dysfunction and 2 had both RV and LV dysfunction. Follow-up echocardiography was available for 89 patients $(67 \%)$. Another six patients had new onset of LV dysfunction at follow-up while eleven had normalized their LV function. In six patients, a new onset of RV dysfunction was found and thirteen others had normalized the RV function. Thus, a total of 42 patients (32\%) had any cardiac dysfunction of whom 18 (14\%) had LV dysfunction, $17(13 \%)$ had RV dysfunction, and 7 (5\%) had both LV and RV dysfunction detected during their stay in the ICU (Fig. 1). Eight patients (6\%) patients with LV dysfunction had LV regional hypokinesia. In two of them, this was attributed to a known history of cardiac disease. Five patients (4\%) had a typical pattern of Takotsubo syndrome with reversible apical and/or midventricular akinesia and no history of cardiac disease. Two patients had suspected COVID-19 myocarditis, and one had the diagnosis verified with cardiac magnetic resonance imaging (MRI). An 18-year-old patient with global LV hypokinesia was diagnosed with paediatric multisystem inflammatory syndrome (PIMS-TS). A cardiac MRI after three months showed mild cardiac fibrosis, consistent with recent myocarditis. 
Patients with LV dysfunction had a larger LV diameter and lower ejection fraction, stroke volume, and cardiac index compared to patients with normal LV function (Table 2). Of the 24 patients with RV dysfunction, 22 had low TAPSE with or without depressed RV function assessed visually, while 2 patients had moderately to severely depressed function assessed visually with preserved TAPSE. Patients with RV dysfunction had more often a tricuspid regurgitation. Elevated PAP was found in 34 patients (26\%). This was diagnosed with a TR Vmax $>2.9$ in 32 patients and indirect criteria in 2 patients. Elevated PAP was more common in patients with RV dysfunction, as compared to patients with normal cardiac function $(58 \%$ vs $22 \% ; p<0.001)$.

Table 2

Echocardiographic data of the 132 participants.

\begin{tabular}{|c|c|c|c|}
\hline \multirow[t]{2}{*}{ Variable } & \multirow{2}{*}{$\begin{array}{l}\text { Normal cardiac function, } \\
n=90\end{array}$} & \multicolumn{2}{|c|}{ Cardiac dysfunction } \\
\hline & & $\begin{array}{l}\text { LV dysfunction, } \\
n=25\end{array}$ & $\begin{array}{l}\text { RV dysfunction, } \\
n=24\end{array}$ \\
\hline LV diameter, mm & $48 \pm 4$ & $52 \pm 6^{*}$ & $49 \pm 5$ \\
\hline LV dysfunction, n (\%) & - & $25(100)^{*}$ & $7(29)^{\star}$ \\
\hline LV ejection fraction, \% & $59 \pm 5$ & $43 \pm 6^{*}$ & $57 \pm 11$ \\
\hline Stroke volume indexed & $40 \pm 12$ & $30 \pm 9 *$ & $37 \pm 11$ \\
\hline Cardiac index & $3,1 \pm 0,9$ & $2.4 \pm 1^{\star}$ & $3.0 \pm 1$ \\
\hline Regional hypokinesia, n (\%) & - & $8(32)^{*}$ & $0(0)$ \\
\hline RV dysfunction, n (\%) & - & $6(24)^{*}$ & $24(100) \star$ \\
\hline TAPSE, mm & $20(19-23)$ & $20(17-22)$ & $15(12-16)^{\star}$ \\
\hline TAPSE < 17, n (\%) & - & $3(12)$ & $22(92)^{\star}$ \\
\hline $\begin{array}{l}\text { Moderate or severe RV } \\
\text { failure, } \mathrm{n}(\%)\end{array}$ & - & $1(4)$ & $8(33)^{\star}$ \\
\hline TR present, n (\%) & $41(46)$ & $15(60)$ & $19(79) *$ \\
\hline TR Vmax, m/s & $3.1(2.4-4.0)$ & $2.7(2.2-3.5)$ & $3.5(2.7-4.5)$ \\
\hline Elevated PAP, n (\%) & $20(22)$ & $6(24)$ & $14(58) *$ \\
\hline
\end{tabular}

$T R$ Vmax is reported from patients with a TR present. $L V=$ left ventricle, $R V=$ right ventricle, TAPSE = tricuspid annular plane systolic excursion; TR = tricuspid regurgitation, TR Vmax = maximal tricuspid regurgitation velocity, $\mathrm{PAP}=$ pulmonary arterial pressure. * $\mathrm{p}$-value $<0.05$ vs group normal cardiac function. In group normal cardiac function, data is reported for first echo. In group LV or RV dysfunction, data is reported for first echo with dysfunction.

\section{Clinical characteristics and routine laboratory data}


Clinical characteristics did not differ between those patients with and those without cardiac dysfunction at the time of the first echocardiography, except that patients with cardiac dysfunction more often had a high dose of noradrenaline $(>0.20 \mu \mathrm{g} / \mathrm{kg} / \mathrm{min})$. In a generalized regression analysis, taking repeated examinations into consideration, a dose of noradrenaline $>0.20 \mu \mathrm{g} / \mathrm{kg} / \mathrm{min}$ was the only clinical variable associated with an increased risk of cardiac dysfunction (see Supplemental data). Levels of d-dimer were slightly higher $(2.0 \mathrm{mg} / \mathrm{L}$ vs $1.6 \mathrm{mg} / \mathrm{L}, \mathrm{p}=0.006)$ in patients with cardiac dysfunction, but there were no differences in other routine laboratory data (Table 3 ). 
Table 3

Hemodynamic, ventilatory data and routine lab at time of first echo

\begin{tabular}{|c|c|c|c|c|}
\hline \multirow[t]{2}{*}{ Category } & \multirow[t]{2}{*}{ Variable } & \multicolumn{2}{|l|}{ Cardiac function } & \multirow{2}{*}{$\begin{array}{l}\mathrm{p}- \\
\text { value }\end{array}$} \\
\hline & & $\begin{array}{l}\text { Normal function } \\
(n=97)\end{array}$ & $\begin{array}{l}\text { Cardiac dysfunction } \\
(n=35)\end{array}$ & \\
\hline \multirow[t]{4}{*}{ Hemodynamic } & $\begin{array}{l}\text { Systolic blood pressure, } \\
\mathrm{mmHg}\end{array}$ & $126 \pm 18$ & $124 \pm 19$ & 0.707 \\
\hline & $\begin{array}{l}\text { Mean arterial pressure, } \\
\mathrm{mmHg}\end{array}$ & $79 \pm 10$ & $78 \pm 12$ & 0.602 \\
\hline & Heart rate, bpm & $78 \pm 15$ & $83 \pm 21$ & 0.138 \\
\hline & $\begin{array}{l}\text { Noradrenaline > } \\
0.20 \mu \mathrm{g} / \mathrm{kg} / \mathrm{min}, \mathrm{n}(\%)\end{array}$ & $7(7)$ & $8(23)$ & 0.025 \\
\hline \multirow[t]{12}{*}{ Ventilatory } & $\mathrm{FiO} 2, \%$ & $50(40-65)$ & $50(45-70)$ & 0.287 \\
\hline & $\mathrm{PaO} 2 / \mathrm{FiO} 2$ & $19(14.6-25.2)$ & $20.6(16.2-24.7)$ & 0.624 \\
\hline & $\begin{array}{l}\text { Mechanical ventilation, } \mathrm{n} \\
(\%)\end{array}$ & $83(86)$ & $31(89)$ & 0.780 \\
\hline & Ventilation mode & & & 0.337 \\
\hline & PCV-VG, n (\%) & $43(44)$ & $14(40)$ & \\
\hline & PSV, n (\%) & $22(23)$ & $5(14)$ & \\
\hline & PCV, n (\%) & $9(9)$ & $8(23)$ & \\
\hline & APRV, n (\%) & $9(9)$ & $4(11)$ & \\
\hline & High flow oxygen, n (\%) & $9(9)$ & $3(9)$ & \\
\hline & NIV, n (\%) & $4(4)$ & $0(0)$ & \\
\hline & Oxygen mask, n (\%) & $1(1)$ & $1(3)$ & \\
\hline & $\begin{array}{l}\text { Prone position within } 24 \\
\text { hours, } \mathrm{n}(\%)\end{array}$ & $36(37)$ & $9(26)$ & 0.299 \\
\hline \multirow[t]{4}{*}{ Routine lab } & Hemoglobin, g/l & $113(105-127)$ & $116(101-122)$ & 0.473 \\
\hline & CRP, mg/L & $230(140-290)$ & $230(190-310)$ & 0.196 \\
\hline & Leukocyte count, per liter & $9.1(7.2-12.2)$ & $9.8(6-13.4)$ & 0.897 \\
\hline & Creatinine, $\mu \mathrm{mol} / \mathrm{L}$ & $81(56-108)$ & $93(66-127)$ & 0.106 \\
\hline
\end{tabular}

$\mathrm{FiO} 2=$ fraction of inspired oxygen, $\mathrm{PaO} 2=$ partial pressure of oxygen, $\mathrm{PEEP}=$ positive end-expiratory pressure, $P C V-V G=$ pressure controlled ventilation - volume guaranteed, $P S V=$ pressure support ventilation, $\mathrm{PCV}=$ pressure controlled ventilation, $\mathrm{APRV}=$ airway pressure release ventilation, NIV = non-invasive ventilation, $\mathrm{CRP}=\mathrm{C}$-reactive protein . 


\begin{tabular}{|c|c|c|c|c|}
\hline \multirow[t]{2}{*}{ Category } & \multirow[t]{2}{*}{ Variable } & \multicolumn{2}{|l|}{ Cardiac function } & \multirow{2}{*}{$\begin{array}{l}\mathrm{p}- \\
\text { value }\end{array}$} \\
\hline & & $\begin{array}{l}\text { Normal function } \\
(n=97)\end{array}$ & $\begin{array}{l}\text { Cardiac dysfunction } \\
(\mathrm{n}=35)\end{array}$ & \\
\hline & Fibrinogen, g/L & $6.6(5.3-7.6)$ & $6.9(5.7-8)$ & 0.557 \\
\hline & D-dimer , mg/L & $1.4(0.82-2.85)$ & $2(1.5-13)$ & 0.006 \\
\hline & Lactate levels, $\mu \mathrm{g} / \mathrm{l}$ & $1.3(1-1.7)$ & $1.4(1.1-1.8)$ & 0.325 \\
\hline \multicolumn{5}{|c|}{$\begin{array}{l}\mathrm{FiO} 2=\text { fraction of inspired oxygen, } \mathrm{PaO} 2=\text { partial pressure of oxygen, } \mathrm{PEEP}=\text { positive end-expiratory } \\
\text { pressure, } \mathrm{PCV}-\mathrm{VG}=\text { pressure controlled ventilation }- \text { volume guaranteed } \mathrm{PSV}=\text { pressure } \text { support } \\
\text { ventilation, } \mathrm{PCV}=\text { pressure controlled ventilation, } \mathrm{APRV}=\text { airway pressure release ventilation, NIV = } \\
\text { non-invasive ventilation, } \mathrm{CRP}=\mathrm{C} \text {-reactive protein. }\end{array}$} \\
\hline
\end{tabular}

\section{Mortality and clinical course}

In total, 24 patients (18\%) died within 30 days of admission to the ICU. Thirty-day mortality was the same in patients with cardiac dysfunction $(n=10,24 \%)$ as in those with normal cardiac function $(n=14,16 \%, p$ $=0.332$ ). There was no increased risk of death at 30 days in patients with as opposed to without cardiac dysfunction in a risk-adjusted model (Odds ratio [OR] 1.56, $p=0.391$ ). RV dysfunction on admission (riskadjusted OR 7.03, $p=0.002$ ), or detected while in the ICU (risk-adjusted OR 3.98, $p=0.013$ ), was associated with an increased risk of death at 30 days. LV dysfunction was not associated with any increased risk of death at 30 days. Elevated PAP detected while in the ICU was associated with an increased risk of death at 30 days (risk-adjusted OR 3.88, $p=0.007$ ) (Table 4). There was also an increased risk of death during the first 90 days from admission in patients with RV dysfunction or elevated PAP (Fig. 2). 
Table 4

Mortality analysis

\begin{tabular}{|c|c|c|c|c|c|c|c|}
\hline \multirow{2}{*}{$\begin{array}{l}\text { Presentation of cardiac } \\
\text { dysfunction }\end{array}$} & \multirow[t]{2}{*}{ Variable } & \multicolumn{3}{|c|}{ Crude risk of death } & \multicolumn{3}{|c|}{ Adjusted risk of death* } \\
\hline & & OR & $\begin{array}{l}95 \% \mathrm{Cl} \\
\text { for OR }\end{array}$ & $\begin{array}{l}\text { p- } \\
\text { value }\end{array}$ & OR & $\begin{array}{l}95 \% \\
\text { Cl for } \\
\text { OR }\end{array}$ & $\begin{array}{l}\mathrm{p} \text { - } \\
\text { value }\end{array}$ \\
\hline On & $\begin{array}{l}\text { Cardiac } \\
\text { dysfunction }\end{array}$ & 1.89 & $\begin{array}{l}0.74- \\
4.83\end{array}$ & 0.182 & 1.56 & $\begin{array}{l}0.56- \\
4.33\end{array}$ & 0.391 \\
\hline \multirow[t]{3}{*}{ admission } & $\begin{array}{l}\text { LV } \\
\text { dysfuncion }\end{array}$ & 0.49 & $\begin{array}{l}0.11- \\
2.26\end{array}$ & 0.358 & 0.22 & $\begin{array}{l}0.04- \\
1.38\end{array}$ & 0.106 \\
\hline & $\begin{array}{l}\text { RV } \\
\text { dysfunction }\end{array}$ & 4.90 & $\begin{array}{l}1.68- \\
14.3\end{array}$ & 0.004 & 7.03 & $\begin{array}{l}2.08- \\
23.8\end{array}$ & 0.002 \\
\hline & Elevated PAP & 2.49 & $\begin{array}{l}0.96- \\
6.45\end{array}$ & 0.061 & 2.34 & $\begin{array}{l}0.84- \\
6.51\end{array}$ & 0.103 \\
\hline At any time & $\begin{array}{l}\text { Cardiac } \\
\text { dysfunction }\end{array}$ & 1.70 & $\begin{array}{l}0.68- \\
4.22\end{array}$ & 0.255 & 1.43 & $\begin{array}{l}0.53- \\
3.83\end{array}$ & 0.476 \\
\hline \multirow[t]{3}{*}{ in ICU } & $\begin{array}{l}\text { LV } \\
\text { dysfuncion }\end{array}$ & 0.56 & $\begin{array}{l}0.15- \\
2.04\end{array}$ & 0.379 & 0.29 & $\begin{array}{l}0.06- \\
1.34\end{array}$ & 0.113 \\
\hline & $\begin{array}{l}\text { RV } \\
\text { dysfunction }\end{array}$ & 3.72 & $\begin{array}{l}1.38- \\
10.0\end{array}$ & 0.009 & 3.98 & $\begin{array}{l}1.34- \\
11.8\end{array}$ & 0.013 \\
\hline & Elevated PAP & 3.92 & $\begin{array}{l}1.57- \\
9.84\end{array}$ & 0.004 & 3.88 & $\begin{array}{l}1.45- \\
10.4\end{array}$ & 0.007 \\
\hline
\end{tabular}

Cardiac dysfunction refers to left or right ventricular dysfunction. $\mathrm{Cl}=$ confidence interval, $\mathrm{OR}=$ odds ratio, $\mathrm{LV}=$ left ventricle, $\mathrm{RV}$ = right venticle, $\mathrm{PAP}=$ pulmonary arterial pressure, $\mathrm{ICU}$ = intensive care unit

* Adjusted for SAPS 3 score

Patients with cardiac dysfunction more often needed continuous renal replacement therapy $(n=16,38 \%)$ as compared to patients with normal cardiac function $(n=16,18 \%, p=0.016)$. Patients with cardiac dysfunction had less days alive outside ICU, as compared to patients with normal cardiac function (median 0 [IQR 0-10] versus 13 days [IQR 0-20], $\mathrm{p}=0.042$ ). Length of hospital stay did not differ between the two groups (see Supplemental data).

\section{Cardiac biomarkers}

Troponin levels were higher in patients with cardiac dysfunction (2.04 times the upper reference levels [IQR 1.06-7.71]) as compared to patients with normal cardiac function (median 0.81 [IQR 0.51-1.64], $p<$ 0.001). Levels of NTproBNP were also higher in patients with cardiac dysfunction (1220 ng/I [IQR 336$3540]$ ), as compared to patients with normal cardiac function (267 ng/I [IQR 139-504], $p<0.001$, Supplemental data). Area under the curve (AUC) of troponin for detection of cardiac dysfunction was 0.729 , and the best cut-off value using Youden's index was troponin $>1.44$ times over the upper reference 
limit. Detection of cardiac dysfunction with NTproBNP had an AUC of 0.744 , with a best cut-off value of a NTproBNP $>857 \mathrm{ng} / \mathrm{l}$. Using a combination of troponin $>1.44$ times the upper reference limit and NTproBNP $>857 \mathrm{ng} / \mathrm{l}$ had a positive predictive value (PPV) of $81 \%$ for detection of cardiac dysfunction. Troponin $<1.44$ times the upper reference limit and NTproBNP $<857 \mathrm{ng} / \mathrm{l}$ had $85 \%$ probability of excluding cardiac dysfunction. (Table 5).

Table 5

Troponin and NTproBNP for detection of cardiac dysfunction

\begin{tabular}{|lllllll}
\hline Test & Variable cut-off & AUC & Sensitivity & Specificity & PPV & NPV \\
\hline $\begin{array}{l}\text { Detection of } \\
\text { cardiac } \\
\text { dysfunction }\end{array}$ & $\begin{array}{l}\text { Troponin }>1.44 \text { times upper } \\
\text { ref }\end{array}$ & 0.729 & $69 \%$ & $72 \%$ & $55 \%$ & $82 \%$ \\
& NTproBNP $>857 \mathrm{ng} / \mathrm{l}$ & 0.744 & $56 \%$ & $89 \%$ & $71 \%$ & $80 \%$ \\
\hline $\begin{array}{l}\text { Troponin }>1.44 \text { times upper } \\
\text { ref and NTproBNP } \\
857 \mathrm{ng} / \mathrm{l}\end{array}$ & $54 \%$ & $94 \%$ & $81 \%$ & $80 \%$ \\
\hline $\begin{array}{l}\text { Exclusion of } \\
\text { cardiac } \\
\text { dysfunction }\end{array}$ & $\begin{array}{l}\text { Troponin }<1.44 \text { times upper } \\
\text { ref and NTproBNP } \\
857 \mathrm{ng} / \mathrm{l}\end{array}$ & $66 \%$ & $76 \%$ & $85 \%$ & $53 \%$ \\
\hline
\end{tabular}

Cardiac biomarkers obtained from echocardiography at first presentation of cardiac dysfunction. In patients with normal echoes, cardiac biomarkers used were also obtained at first echocardiography. $\mathrm{AUC}=$ area under curve, $\mathrm{PPV}=$ positive predictive value, $\mathrm{NPV}=$ negative predictive value, $\mathrm{NTproBNP}=$ $\mathrm{N}$-terminal pro b-type natriuretic peptide

\section{Discussion}

The main findings of this study are that (1) cardiac dysfunction commonly occurs in critical illness caused by COVID-19; (2) RV dysfunction, defined as a TAPSE $<17 \mathrm{~mm}$ or moderately to severely depressed function assessed visually, was associated with an increased risk of death; (3) cardiac dysfunction is not easily recognized by clinical variables; and that (4) cardiac biomarkers have a moderate value in detecting cardiac dysfunction in patients critically ill with COVID-19.

We found that $32 \%$ of the patients had LV or RV dysfunction at some time during their stay in the ICU. Cardiac dysfunction was more common at the time of admission to the ICU than later in the ICU-period. The incidence of cardiac dysfunction was in line with other studies of patients hospitalized, whose COVID-19 disease severities ranged from mild to severe, and where LV dysfunction was found in 10 to $42 \%$ and RV dysfunction in 14 to $39 \%[15,23,24]$. One reason for the relatively low incidence of RV dysfunction may have been the liberal use of thromboprophylaxis that was introduced early in the study period [25], leading to less pulmonary embolism than in many earlier studies [15, 26]. We did not assess patients with tissue doppler or strain analysis, modalities that are more sensitive for the detection of ventricular dysfunction, which could explain a comparative low incidence of cardiac dysfunction in our study. 
We found a number of different types of LV dysfunction. Two patients had suspected COVID-19 myocarditis; the diagnosis was verified in one patient. Knowledge of COVID-19 myocarditis is still very limited. Patients are reported to present in various ways and they may have either global or regional hypokinesia, with or without preserved ejection fraction, and their recovery time may be brief or prolonged $[27,28]$. There are no strict criteria for COVID-19 myocarditis. In the present study we can neither confirm nor exclude additional cases within our cohort. Five patients presented with a clinical presentation of the Takotsubo syndrome. Severe respiratory distress is an established trigger of Takotsubo, and the incidence we found is in agreement with other studies of Takotsubo syndrome in critically ill patients [29]. Moreover, Takotsubo has also been reported in several case studies of patients with COVID-19 [11, 12]. One patient was diagnosed with PIMS-TS, a condition associated with cardiac dysfunction in COVID-19 [30]. Other plausible causes of LV dysfunction are secondary effects due to hypoxia, hypotension, or a toxic effect due to the inflammatory state [31]. RV dysfunction was seen in $18 \%$ of participants, and there was a close correlation between elevated PAP and RV dysfunction. Thus, RV dysfunction in most cases is probably attributable to an increased RV afterload. Elevated PAP was common in our study population, having been seen in nearly one-third of all patients. Both pulmonary embolism and pulmonary microangiopathy with microthrombosis are common in severe cases of COVID-19 and are known causes of elevated PAP $[32,33]$. Pulmonary hypoxia with hypoxic vasoconstriction was another likely cause of elevated PAP among our study patients [34]. Increased right chamber afterload due to mechanical ventilation is of course a common cause of elevated PAP in ICU patients in general.

Cardiac dysfunction was not associated with an increased risk of death in our study. However, patients with cardiac dysfunction had a more complex course of disease with a greater need for renal replacement therapy and less ICU-free days. It is unclear if this is a causal relationship, or if cardiac dysfunction is a marker for more severe disease. In a sub-group analysis, RV dysfunction and elevated PAP were independently associated with an increased risk of death. This is consistent with findings from other studies of patients with ARDS and hospitalized patients with COVID-19 [13, 14]. Regrettably, the main cause of death was not registered in the present study, and patients were not systematically assessed for pulmonary embolism. It is, therefore, unclear if RV dysfunction and elevated PAP are only markers of more severe pulmonary disease, with a subsequent risk of pulmonary collapse and respiratory death, or if there are other direct causes as well. It seems reasonable to assume that patients with RV dysfunction, elevated PAP, or both will benefit from further investigation by computer tomography for diagnosis of pulmonary embolism, worsening of ARDS, COVID-19 typical infiltrates, or secondary bacterial infection. Furthermore, pulmonary vasodilators could be tried to see if there is improvement in RV function or decreased PAP [35]. In severe cases of COVID-19, RV dysfunction could be supportive in a decision to prepare for, or initiate, extracorporeal membrane oxygenation (ECMO).

Cardiac dysfunction was not easily detected by clinical characteristics. Our hypothesis was that cardiac dysfunction would be associated with a more severe clinical picture, such as higher levels of oxygen, respiratory support, or elevated lactate levels. The only variable associated with an increased risk of having cardiac dysfunction was a high dose of noradrenaline. It is likely because that critical illness from COVID-19 is such a severe respiratory disease, the contribution of cardiac dysfunction is not detectable by 
clinical variables in such a patient population. However, high doses of noradrenaline should be considered a marker for a more severe cardiovascular deterioration. In patients requiring noradrenaline > $0.20 \mu \mathrm{g} / \mathrm{kg} / \mathrm{min}$, cardiac dysfunction should be suspected and echocardiography ought to be performed.

The cardiac biomarkers troponin and NTproBNP only showed a moderate ability to detect cardiac dysfunction. However, a combination of troponin at less than 1.44 times its upper reference limit and NTproBNP $<857 \mathrm{ng} / \mathrm{l}$ had a negative predictive value of $85 \%$ of excluding cardiac dysfunction and might be used as a tool to rule out the need of echocardiography. This could be important in ICUs treating patients with COVID-19, where there is a need to conserve personal protective equipment and minimize the number of individuals interacting with contagious patients. On the other hand, levels above these limits had a predictive value of $81 \%$ for detection of cardiac dysfunction, suggesting that echocardiography should be performed.

Our study has some limitations. Inclusion rates were relatively low, as only $40 \%$ of the potential study population was included, mostly due to a lack of study resources. Moreover, a number of patients were retrospectively included. Nevertheless, a sensitivity analysis showed the study population was a representative sample of each site's total population. The main strength of the study is the multi-centre design. Although the sample size is relatively low in absolute numbers, it is the largest study to date with echocardiographic evaluation, cardiac biomarkers, and clinical data carried out among COVID-19 patients in an ICU-setting.

\section{Conclusions}

Cardiac dysfunction is common in critical illness caused by COVID-19, but it is not easily detected on the basis of clinical characteristics. Cardiac biomarkers here prove be helpful. The ominous sign of RV dysfunction benefit from further investigation and treatment with pulmonary vasodilators or initiation of ECMO.

\section{Abbreviations}

APRV

airway pressure release ventilation

ARDS

acute respiratory distress syndrome

AUC

area under curve

$\mathrm{BMI}$

body mass index

COPD

chronic, obstructive pulmonary disease

CRP 
C-reactive protein

CRRT

continuous renal replacement therapy

ECMO

extracorporeal membrane oxygenation

ICU

intensive care unit

IQR

interquartile range

LMWH

low molecular weight heparine

LV

left ventricular

LVOT

area of LV outflow tract

MRI

magnetic resonance imaging

NIV

non-invasive ventilation,

NTproBNP

$\mathrm{N}$-terminal pro b-type natriuretic peptide

OR

odds ratio

PAP

pulmonary artery pressure

PEEP

positive end-expiratory pressure

PCV

pressure controlled ventilation

PCV-VG

pressure controlled ventilation - volume guaranteed

PIMS-TS

paediatric multisystem inflammatory syndrome

PPV

positive predictive value

PSV

pressure support ventilation

RAAS-blockers

renin angiotensin aldosterone system blockers

ROC 
receiver operating characteristics

RV

right ventricular

SAPS 3

simplified acute physiology score 3

SARS-CoV-2

severe acute respiratory syndrome coronavirus 2

TAPSE

tricuspid annular plane systolic excursion

TR

tricuspid regurgitation

TR Vmax

jet velocity of a tricuspid regurgitation

VTI

velocity time integral

$95 \% \mathrm{Cl}$

$95 \%$ confidence interval

\section{Declarations}

\section{Ethics approval and consent to participate}

This study was approved by the Swedish Ethics Review Authority Sweden (approval number 036-18, 2020-01684 and 2020-03815). Consent to be included in the study was obtained from the patient or patient's next of kin whenever possible. For patients retrospectively included, patients consent was waived.

Consent for publication

No individual data was reported in the study.

Availability of data and materials

The datasets used and/or analysed during the current study are available from the corresponding author on reasonable request.

Competing interests

$\mathrm{JO}$ and BR received funding from the Swedish Heart-Lung foundation for the conduct of this study. The other authors declare that they have no competing interests.

Funding 
The study was funded by the Swedish Heart-Lung foundation (number 20200253 and 2017063).

\section{Authors' contributions}

$\mathrm{JH}$ initation of the study, conceptualization and study design, drafting and revising the manuscript, echocardiographic examinations, data acquisition and interpretation. JBF study design, inclusion of patients, data acquisition, compilation and interpretation, drafting and revising the manuscript. CJ study design, echocardiographic examinations, data acquisition. KD, NN echocardiographic examinations, data acquisition. SM, JC data acquisition. ERW data acquisition and compilation. OC reviewing echocardiographic examinations, data acquisition. AY, study design, substantively revised the manuscript. MC data acquisition, substantively revised the manuscript. BR study design and conceptualization, statistical analyses, funding. JO initiation of the study, conceptualization and study design, echocardiographic examinations, data acquisition and interpretation, statistical analyses, funding, drafting and revising the manuscript. All authors read and revised the paper. All authors have agreed to be accountable for all aspects of the work. All authors approved the final version of the manuscript.

\section{Acknowledgements}

Not applicable

\section{References}

\section{World. Health Organization; Coronavirus disease (COVID-19) Situation Report - 112} https://www.who.int/emergencies/diseases/novel-coronavirus-2019/situation-reports Accessed 21 December.

2. Wu Z, McGoogan JM. Characteristics of and Important Lessons From the Coronavirus Disease 2019 (COVID-19) Outbreak in China: Summary of a Report of 72314 Cases From the Chinese Center for Disease Control and Prevention. JAMA. 2020;323(13):1239-42.

3. Oran DP, Topol EJ. Prevalence of Asymptomatic SARS-CoV-2 Infection: A Narrative Review. Ann Intern Med. 2020;173(5):362-7.

4. Wang D, Hu B, Hu C, Zhu F, Liu X, Zhang J, Wang B, Xiang H, Cheng Z, Xiong Y, et al. Clinical Characteristics of 138 Hospitalized Patients With 2019 Novel Coronavirus-Infected Pneumonia in Wuhan, China. JAMA. 2020;323(11):1061-9.

5. Arcari L, Luciani M, Cacciotti L, Musumeci MB, Spuntarelli V, Pistella E, Martolini D, Manzo D, Pucci M, Marone $\mathrm{C}$, et al. Incidence and determinants of high-sensitivity troponin and natriuretic peptides elevation at admission in hospitalized COVID-19 pneumonia patients. Intern Emerg Med. 2020;15(8):1467-76.

6. Huang C, Wang Y, Li X, Ren L, Zhao J, Hu Y, Zhang L, Fan G, Xu J, Gu X, et al. Clinical features of patients infected with 2019 novel coronavirus in Wuhan, China. Lancet. 2020;395(10223):497-506.

7. Xie J, Wu W, Li S, Hu Y, Hu M, Li J, Yang Y, Huang T, Zheng K, Wang Y, et al. Clinical characteristics and outcomes of critically ill patients with novel coronavirus infectious disease (COVID-19) in China: 
a retrospective multicenter study. Intensive Care Med. 2020;46(10):1863-72.

8. Inciardi RM, Lupi L, Zaccone G, Italia L, Raffo M, Tomasoni D, Cani DS, Cerini M, Farina D, Gavazzi E, et al: Cardiac Involvement in a Patient With Coronavirus Disease 2019 (COVID-19). JAMA Cardiol 2020, 5(7):819-824.

9. Sala S, Peretto G, Gramegna M, Palmisano A, Villatore A, Vignale D, De Cobelli F, Tresoldi M, Cappelletti AM, Basso $\mathrm{C}$, et al. Acute myocarditis presenting as a reverse Tako-Tsubo syndrome in a patient with SARS-CoV-2 respiratory infection. Eur Heart J. 2020;41(19):1861-2.

10. Deng Q, Hu B, Zhang Y, Wang H, Zhou X, Hu W, Cheng Y, Yan J, Ping H, Zhou Q. Suspected myocardial injury in patients with COVID-19: Evidence from front-line clinical observation in Wuhan, China. Int J Cardiol. 2020;311:116-21.

11. Meyer P, Degrauwe S, Van Delden C, Ghadri JR, Templin C. Typical takotsubo syndrome triggered by SARS-CoV-2 infection. Eur Heart J. 2020;41(19):1860.

12. Dweck MR, Bularga A, Hahn RT, Bing R, Lee KK, Chapman AR, White A, Salvo GD, Sade LE, Pearce K, et al. Global evaluation of echocardiography in patients with COVID-19. Eur Heart J CardiovasC Imaging. 2020;21(9):949-58.

13. Liu Y, Xie J, Gao P, Tian R, Qian H, Guo F, Yan X, Song Y, Chen W, Fang L, et al: Swollen heart in COVID19 patients who progress to critical illness: a perspective from echo-cardiologists. ESC Heart Fail 2020.

14. Lassen MCH, Skaarup KG, Lind JN, Alhakak AS, Sengelov M, Nielsen AB, Espersen C, Ravnkilde K, Hauser R, Schops LB, et al: Echocardiographic abnormalities and predictors of mortality in hospitalized COVID-19 patients: the ECHOVID-19 study. ESC Heart Fail 2020.

15. Szekely Y, Lichter Y, Taieb P, Banai A, Hochstadt A, Merdler I, Gal Oz A, Rothschild E, Baruch G, Peri Y, et al. Spectrum of Cardiac Manifestations in COVID-19: A Systematic Echocardiographic Study. Circulation. 2020;142(4):342-53.

16. Li Y, Li H, Zhu S, Xie Y, Wang B, He L, Zhang D, Zhang Y, Yuan H, Wu C, et al. Prognostic Value of Right Ventricular Longitudinal Strain in Patients With COVID-19. JACC Cardiovasc Imaging. 2020;13(11):2287-99.

17. Lazzeri C, Bonizzoli M, Batacchi S, Cianchi G, Franci A, Fulceri GE, Peris A. Cardiac Involvment in COVID-19-Related Acute Respiratory Distress Syndrome. Am J Cardiol. 2020;132:147-9.

18. Baycan OF, Barman HA, Atici A, Tatlisu A, Bolen F, Ergen P, Icten S, Gungor B, Caliskan M. Evaluation of biventricular function in patients with COVID-19 using speckle tracking echocardiography. Int $J$ Cardiovasc Imaging 2020:1-10.

19. Galiè N, Humbert M, Vachiery J-L, Gibbs S, Lang I, Torbicki A, Simonneau G, Peacock A, Vonk Noordegraaf A, Beghetti M, et al. 2015 ESC/ERS Guidelines for the diagnosis and treatment of pulmonary hypertension: The Joint Task Force for the Diagnosis and Treatment of Pulmonary Hypertension of the European Society of Cardiology (ESC) and the European Respiratory Society (ERS): Endorsed by: Association for European Paediatric and Congenital Cardiology (AEPC), International Society for Heart and Lung Transplantation (ISHLT). Eur Heart J. 2015;37(1):67-119. 
20. Parasuraman S, Walker S, Loudon BL, Gollop ND, Wilson AM, Lowery C, Frenneaux MP. Assessment of pulmonary artery pressure by echocardiography-A comprehensive review. IJC Heart Vasculature. 2016;12:45-51.

21. Metnitz PG, Moreno RP, Almeida E, Jordan B, Bauer P, Campos RA, lapichino G, Edbrooke D, Capuzzo M, Le Gall JR. SAPS 3-From evaluation of the patient to evaluation of the intensive care unit. Part 1: Objectives, methods and cohort description. Intensive Care Med. 2005;31(10):1336-44.

22. Data portal for The Swedish Intensive Care Registry http://portal.icuregswe.org/utdata/en/home.

23. Jain SS, Liu Q, Raikhelkar J, Fried J, Elias P, Poterucha TJ, DeFilippis EM, Rosenblum H, Wang EY, Redfors B, et al. Indications for and Findings on Transthoracic Echocardiography in COVID-19. J Am Soc Echocardiogr. 2020;33(10):1278-84.

24. Li Y, Li H, Li M, Zhang L, Xie M. The prevalence, risk factors and outcome of cardiac dysfunction in hospitalized patients with COVID-19. Intensive Care Med. 2020;46(11):2096-8.

25. Jonmarker S, Hollenberg J, Dahlberg M, Stackelberg O, Litorell J, Everhov ÅH, Järnbert-Pettersson H, Söderberg M, Grip J, Schandl A, et al. Dosing of thromboprophylaxis and mortality in critically ill COVID-19 patients. Crit Care. 2020;24(1):653.

26. Klok FA, Kruip MJHA, van Der Meer NJM, Arbous MS, Gommers D, Kant KM, Kaptein FHJ, van Paassen J, Stals MAM, Huisman MV, et al: Confirmation of the high cumulative incidence of thrombotic complications in critically ill ICU patients with COVID-19: An updated analysis. Thrombosis research 2020.

27. Sawalha K, Abozenah M, Kadado AJ, Battisha A, Al-Akchar M, Salerno C, Hernandez-Montfort J, Islam AM. Systematic review of COVID-19 related myocarditis: Insights on management and outcome. Cardiovasc Revasc Med. 2020;S1553-8389(1520):30497-8.

28. Kariyanna PT, Sutarjono B, Grewal E, Singh KP, Aurora L, Smith L, Chandrakumar HP, Jayarangaiah A, Goldman SA, Salifu MO, et al. A Systematic Review of COVID-19 and Myocarditis. Am J Med Case Rep. 2020;8(9):299-305.

29. Oras J, Lundgren J, Redfors B, Brandin D, Omerovic E, Seeman-Lodding H, Ricksten SE. Takotsubo syndrome in hemodynamically unstable patients admitted to the intensive care unit - a retrospective study. Acta anaesthesiologica Scandinavica. 2017;61(8):914-24.

30. Jiang L, Tang K, Levin M, Irfan O, Morris SK, Wilson K, Klein JD, Bhutta ZA. COVID-19 and multisystem inflammatory syndrome in children and adolescents. Lancet Infect Dis. 2020;20(11):e276-88.

31. Puntmann VO, Carerj ML, Wieters I, Fahim M, Arendt C, Hoffmann J, Shchendrygina A, Escher F, VasaNicotera M, Zeiher AM, et al: Outcomes of Cardiovascular Magnetic Resonance Imaging in Patients Recently Recovered From Coronavirus Disease 2019 (COVID-19). JAMA Cardiology 2020, 5(11):1265-1273.

32. Ackermann M, Verleden SE, Kuehnel M, Haverich A, Welte T, Laenger F, Vanstapel A, Werlein C, Stark $\mathrm{H}$, Tzankov A, et al. Pulmonary Vascular Endothelialitis, Thrombosis, and Angiogenesis in Covid-19. N Engl J Med. 2020;383(2):120-8. 
33. Roncon L, Zuin M, Barco S, Valerio L, Zuliani G, Zonzin P, Konstantinides SV. Incidence of acute pulmonary embolism in COVID-19 patients: Systematic review and meta-analysis. European journal of internal medicine 2020.

34. Ryan D, Frohlich S, McLoughlin P. Pulmonary vascular dysfunction in ARDS. Ann Intensive Care. 2014;4:28-8.

35. Price LC, Wort SJ, Finney SJ, Marino PS, Brett SJ. Pulmonary vascular and right ventricular dysfunction in adult critical care: current and emerging options for management: a systematic literature review. Crit Care (London England). 2010;14(5):R169-9.

\section{Figures}


344 Patients Admitted to

Participating Centers During

Study Period

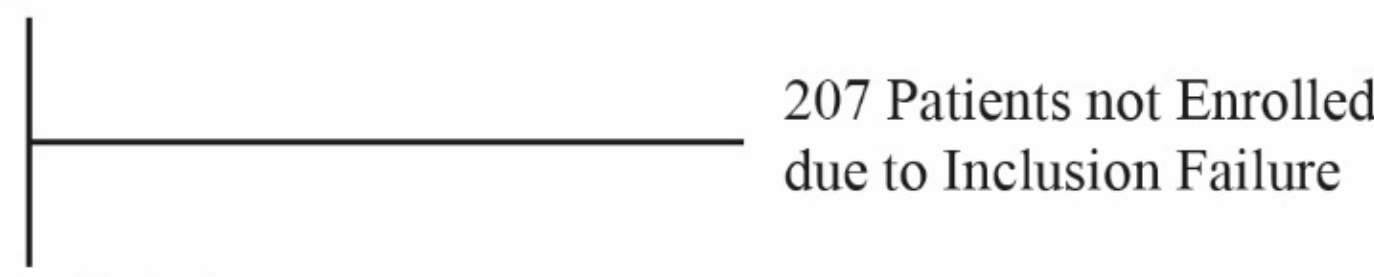

137 Patients Included

in the Study

132 Patients Included in

Final Analysis

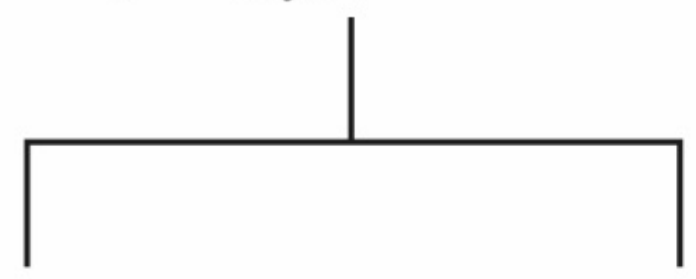

90 Patients with

Normal Cardiac

Function
42 Patients with

Cardiac Dysfunction

- 35: Dysfunction Detected on Admission

- 7: Dysfunction Detected later in ICU

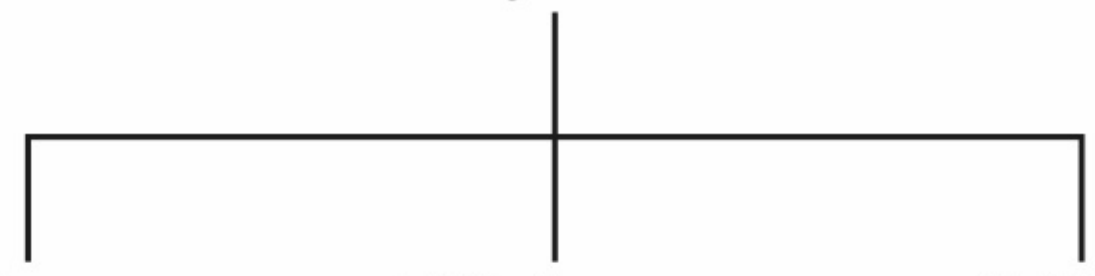

7 Patients:

LV and RV

Dysfunction
5 Patients Excluded:

- 2 as $>72$ Hours Elapsed from Admission to Echo

- 3 Due to Poor Echo Image Quality 

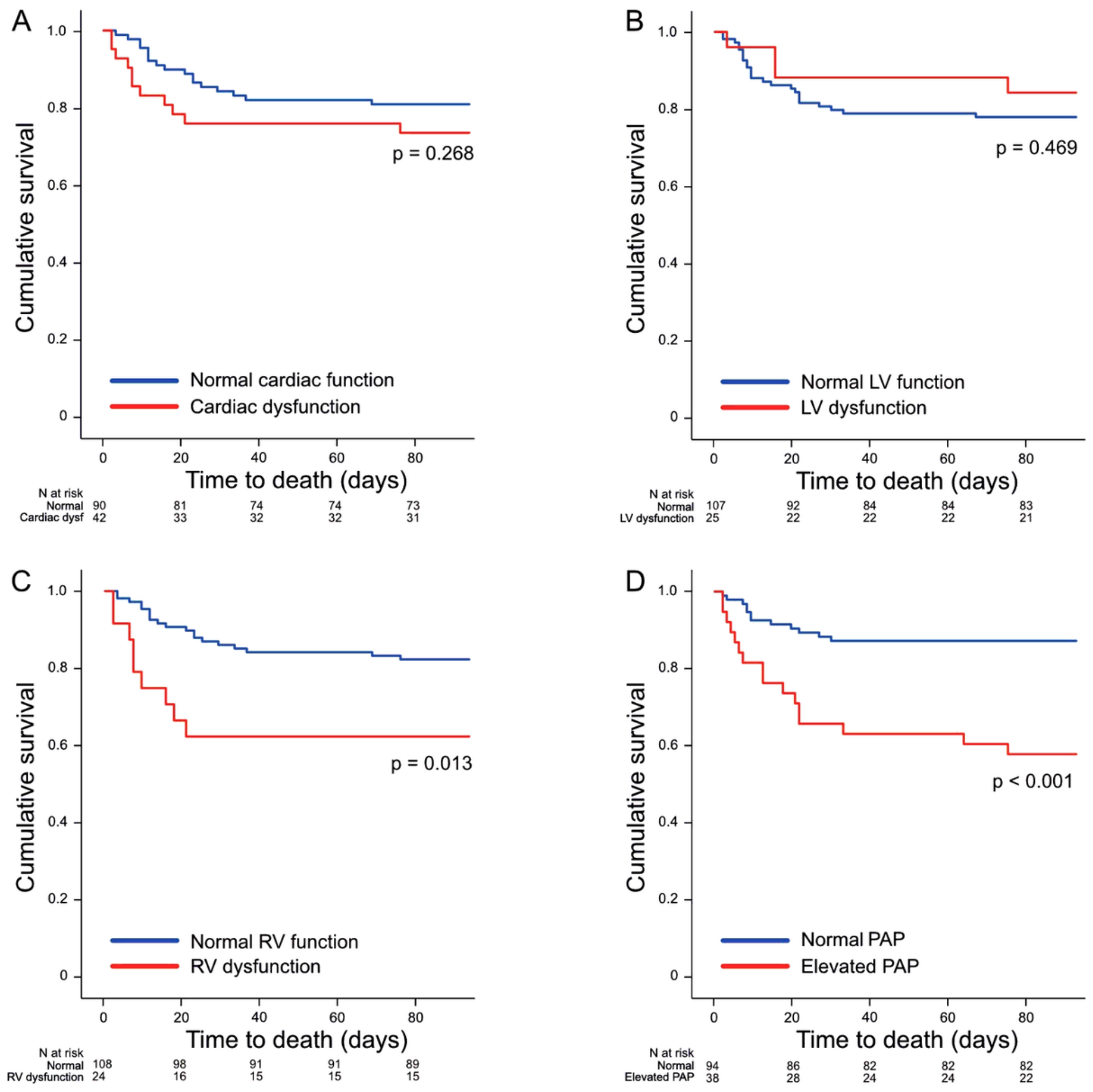

Figure 2

Kaplan-Meier survival curves for patients with versus without cardiac dysfunction (A), LV dysfunction (B), RV dysfunction (C) and elevated PAP (D). The time is calculated from first echo, or in patients with presentation of dysfunction later in the ICU period, the time of second echo. LV = left ventricle, RV = right ventricle

\section{Supplementary Files}


This is a list of supplementary files associated with this preprint. Click to download.

- Supplementaldata.docx 